\title{
EFFECTS OF STATIC MAGNETIC FIELDS ON SUPERCOOLING AND FREEZING KINETICS OF PURE WATER AND 0.9\% NaCI SOLUTIONS
}

\author{
Laura Otero $^{1}$, Antonio C. Rodríguez, and Pedro D. Sanz \\ Institute of Food Science, Technology and Nutrition (ICTAN-CSIC). c/ José Antonio Novais, 10, \\ 28040 Madrid, Spain
}

\section{ABSTRACT}

Previous papers in the literature show no agreement on the effects of static magnetic fields (SMFs) on water supercooling and freezing kinetics. Hypothetical effects of the SMF orientation and the presence of ions in the sample are also unclear. To shed light on this matter, we froze 10-mL pure water samples and $0.9 \% \mathrm{NaCl}$ solutions subjected or not to the SMFs generated by two magnets. We found that the relative position of the magnet poles affected the magnetic field orientation, strength, and the spatial magnetic gradients established throughout the sample. Thus, the SMF strength ranged from 107 to $359 \mathrm{mT}$ when unlike magnet poles faced each other whereas it ranged from 0 to $241 \mathrm{mT}$ when like magnet poles were next to each other. At both conditions, we did not detect any effect of the SMFs on the time at which nucleation occurred, the extent of supercooling, and the phase transition and total freezing times in both pure water and $0.9 \% \mathrm{NaCl}$ solutions. More experiments, under well-characterized SMFs, should be performed to definitively evaluate the ability of SMFs in improving food freezing.

Keywords: static magnetic fields; spatial magnetic gradients; supercooling; freezing kinetics; water; chloride sodium solutions

\section{INTRODUCTION}

Static magnetic fields (SMFs) can visibly affect water. For example, water droplets can levitate in air when they are in a magnetic field of $10 \mathrm{~T}$ or higher (Beaugnon and Tournier, 1991; Ikezoe et al., 1998). Weaker SMFs of the order of one third of a tesla can still produce a $0.25-\mu \mathrm{m}$ depression in the water surface (Chen and Dahlberg, 2011). At these conditions, some water properties such as the viscosity, the surface tension force, or the refractive index, among others, seem to be affected (Cai et al., 2009; Hosoda et al., 2004; Pang et al., 2012; Pang and

\footnotetext{
${ }^{1}$ Corresponding author: Tel.: +34 9154923 00; fax: +34 915493627 .

E-mail address: I.otero@ictan.csic.es (L. Otero).
} 
Deng, 2008a, b; Toledo et al., 2008), but the experimental data published in the literature generally have low reproducibility and little consistency.

The mechanisms explaining the effects of SMFs on water properties are not clear (Otero et al., 2016). Most theories conclude that SMFs affect the hydrogen-bond networks, but there is no agreement on how they are affected. Some authors claim that SMFs cause the weakening of hydrogen bonds (Wang et al., 2013; Zhou et al., 2000), whereas other researchers consider that SMFs enhance the bonding among water molecules (Chang and Weng, 2006). Rearragements in hydrogen bonding can substantially affect the interactions between water molecules and, consequently, impact on some water properties that govern kinetics of some processes such as freezing or vaporization (Szcześ et al., 2011; Toledo et al., 2008). In this sense, Inaba et al. (2004) found that exposition to $6 \mathrm{~T}$ increased the freezing point of water by $5.6 \times 10^{-3}{ }^{\circ} \mathrm{C}$ and, therefore, they concluded that SMFs strengthened hydrogen bonding between water molecules.

In recent years, the ability of static and/or oscillating magnetic fields to improve food freezing has been investigated by many research groups (Erikson et al., 2016; James et al., 2015; Lou et al., 2013; Otero et al., 2017) and some patents have been developed and commercially implemented (Owada, 2007; Owada and Saito, 2010; Sato and Fujita, 2008). It is generally assumed that the application of magnetic fields during freezing inhibits ice nucleation and allows the product to remain largely supercooled, that is, unfrozen at a temperature well below its freezing point. A tentative explanation for this behavior could be an enhancement of $\mathrm{H}$-bonding produced by SMFs. Thus, findings reported by Senesi et al. (2013) suggest that the strengthening of hydrogen bonding can favor water supercooling. It is well-known that the greater the extent of supercooling attained before nucleation, the larger the amount of ice instantaneously formed when nucleation occurs and, consequently, the shorter the phase transition time and the smaller the size of the ice crystals (Zaritzky, 2011). Small ice crystals reduce cellular damage and quality losses in frozen food (Petzold and Aguilera, 2009; Zaritzky, 2011). Therefore, if the application of SMFs during freezing were effective in increasing supercooling, it could be an interesting strategy for improving food freezing. Moreover, SMFs are also supposed to impact on some water properties that govern freezing kinetics such as the freezing point, the internal energy, or the specific heat of water (Inaba et al., 2004; Pang et al., 2012; Zhou et al., 2000) and, therefore, some effects of SMFs on freezing times should also be expected.

However, the experimental data reported in the literature do not give clear evidence of the effects of SMFs on either water supercooling or freezing kinetics. Thus, when freezing water under SMFs, Zhou et al. (2012) observed that supercooling increased with the SMF intensity (up to $5.95 \mathrm{mT}$ ); Aleksandrov et al. (2000) noted the opposite, that is, supercooling decreased when increasing the SMF strength (71-505 mT) whereas Zhao et al. (2017) did not detect any SMF effect (0-43.5 mT) on either supercooling or the phase transition time. Nevertheless, when freezing $5-\mathrm{mL} 0.9 \% \mathrm{NaCl}$ samples, these latter authors found that SMFs enhanced supercooling and reduced the phase transition time by about $55 \%$. They suggested that an 
enhanced mobility of $\mathrm{Na}^{+}$and $\mathrm{Cl}^{-}$ions under SMFs could be responsible for a larger thermal diffusion coefficient and, consequently, for a shorter phase transition time. However, their results differ from those reported by Mok et al. (2015) who also froze $2-\mathrm{mL} 0.9 \% \mathrm{NaCl}$ samples between two neodymium magnets. Depending on the magnets arrangement, the phase transition time increased by $17 \%$ ( $480 \mathrm{mT}$, unlike magnet poles faced each other: attractive position) or reduced by $32 \%$ ( $50 \mathrm{mT}$, like magnet poles faced each other: repulsive position) compared with the control. Therefore, the authors concluded that the direction of the field forces might play a relevant role in the freezing process.

The comparison of the results obtained by different laboratories is often difficult due to two major reasons. On the one hand, the SMFs actually applied in the experiments are frequently not reported rigorously and the spatial magnetic gradients established throughout the sample are completely ignored. On the other hand, the number of replicated experiments sometimes is insufficient to capture the stochastic nature of ice nucleation and the statistics are unclear. Therefore, there is an urgent need to perform well-defined experiments that can be replicated and confirmed by different laboratories. To do so, the SMFs applied to the sample should be characterized accurately and carefully controlled. When assessing the effects of SMFs on supercooling, enough number of freezing experiments with and without SMF application should be replicated to characterize the probability functions correctly. Furthermore, when comparing freezing kinetics, the sample size and the cooling rate should be adjusted so that differences in the duration of the characteristic steps of the freezing process can be easily detected. Moreover, when assessing the efficacy of SMFs in improving food freezing, the sample size should be appropriate to exhibit the spatial magnetic and thermal gradients established in real foods during freezing. In this case, the temperature evolution should be recorded not only at the sample center, as is usual in the literature, but also at the surface. Otherwise, the detection of the exact time at which nucleation occurs is difficult due to the thermal gradients that are established throughout the sample.

To give evidence of the effects of static magnetic fields on water supercooling and freezing kinetics, we performed freezing experiments with 10-mL pure water samples subjected or not to the SMFs generated by two magnets. We designed and constructed a device for holding both the sample and the magnets and ensuring identical SMFs in repeated experiments. To study any hypothetical effect of the direction of the field forces, the magnets were arranged either in attractive or in repulsive position in different experiments. The SMFs generated in each condition were characterized by solving the Maxwell's equations that define the magnetostatic problem. Experimental SMF measurements were then performed to corroborate the modeled results. During the freezing experiments, we recorded the temperature evolution at the sample center and the surface. The freezing curves were then analyzed to obtain some characteristic parameters representing the main steps of the freezing process; namely, the time at which nucleation occurred, the temperature at the sample center when nucleation was triggered, the extent of supercooling attained at the sample center, and the phase transition and total freezing times. To study any effect due to the presence of ions in the sample, freezing experiments were 
113 This paper provides reliable data, collected under easily reproducible conditions, for evaluating 114 the effects of SMFs on supercooling and freezing kinetics. In this way, it increases the 115 knowledge on the ability that magnetic fields have to improve food freezing.

\section{MATERIALS AND METHODS}

\subsection{Samples}

120 Ultrapure water (type I, Milli-Q system, Millipore, Billerica, MA, USA) and $0.9 \% \mathrm{NaCl}$ (Sigma121 Aldrich Corp., St. Louis, MO, USA) solutions in ultrapure water were used in this study. Before 122 each experiment, $10 \mathrm{~mL}$ of freshly prepared sample (samples were not reused) was located in a 123 12-mL glass vial (outer diameter: $23.2 \mathrm{~mm}$, height: $38.1 \mathrm{~mm}$ ) and tempered in a thermostatic bath for, at least, 60 min to achieve a uniform temperature of $25 \pm 0.5^{\circ} \mathrm{C}$.

\subsection{Freezing experiments}

127 Freezing experiments were performed by immersing the sample in a thermostatic bath (model 128 Haake F3-K, Fisons Instruments, Inc., Saddle Brook, NJ, USA) filled with ethanol and maintained at $-25 \pm 0.2{ }^{\circ} \mathrm{C}$. The samples were frozen at different conditions, both with and without SMF application.

In SMF experiments, two neodymium magnets (diameter: $35 \mathrm{~mm}$, height: $20 \mathrm{~mm}$ ) axially magnetized (S-35-20-N, Webcraft GmbH, Gottmadingen, Germany) were employed to generate different static magnetic fields. A device was specially designed and fabricated for holding both the sample and the magnets at fixed positions and, thus, ensuring identical SMFs in repeated experiments (Fig. 1). Basically, it consisted of two blocks of polymethyl methacrylate (PMMA), $80 \mathrm{~mm} \times 80 \mathrm{~mm} \times 28 \mathrm{~mm}$, joined by four Teflon ${ }^{\circledR}$ bolts. Both PMMA blocks had a cylindrical blind hole to lodge the magnets and two removable PMMA lids ( $80 \mathrm{~mm} \times 80 \mathrm{~mm} \times 3 \mathrm{~mm}$ ) that allowed the magnet manipulation to change the relative position of their poles. The sample was located on a glass support between the magnets in such a way that the sample center was equidistant and aligned with the geometric center of both magnets. In all the freezing experiments, the distance between the PMMA blocks was set at $32 \mathrm{~mm}$; that is, the distance that allowed obtaining the maximum field intensity at the conditions tested. To test any hypothetical effect of the direction of the field forces, the magnet poles were placed in attractive or repulsive positions; that is, with unlike or like poles faced each other, in SMF-A and SMF-R experiments, respectively. A similar device, but with solid PMMA blocks (that is, with no holes and lids to lodge magnets), was employed to hold the sample in control experiments with no

147 SMF application. 
Before the experiments, the sample holder was immersed in the cooling medium at $-25^{\circ} \mathrm{C}$ for, at least, $30 \mathrm{~min}$. Once the system was tempered, the sample was placed on the glass support between the PMMA blocks and the freezing experiment started. During the experiments, three T-type thermocouples were employed to measure the temperature at the geometric center of the sample, the glass-vial surface, and the cooling medium. For each condition tested, freezing curves were obtained from the temperature data at the sample center. The temperature at the glass-vial surface was used to detect the time at which nucleation occurred, while the temperature of the cooling medium was monitored to verify that it remained constant during the experiment. All thermocouple measurements were recorded every second by a data acquisition system (DAQMaster MW100, Yokogawa, Tokyo, Japan). Freezing experiments were considered finished when the sample center reached $-20^{\circ} \mathrm{C}$. All the freezing experiments were independently repeated thirty times.

\subsection{Characterization of the static magnetic fields applied during freezing experiments}

The SMFs generated between the magnets, arranged either in attractive or in repulsive position, were modeled and simulated by using the commercial software COMSOL Multiphysics ${ }^{\circledR}$ (v. 4.2, COMSOL AB, Stockholm, Sweden) and the corresponding AC/DC Module. The computational domain included the magnets, the liquid sample, and the air between them. Other components made with non-magnetic materials, such as the PMMA blocks, the Teflon ${ }^{\circledR}$ bolts and nuts, the glass vial and support, and the plastic cap of the sample vial were not taken into account. Due to symmetry, only one quarter of the entire domain was modeled.

Simulations were performed using the finite element (FE) method to solve the Maxwell's equations that define the magnetostatic problem:

$$
\begin{aligned}
\nabla \times \vec{H} & =\overrightarrow{0} \\
\nabla \cdot \vec{B} & =0
\end{aligned}
$$

where $\vec{H}$ represents the magnetic field intensity and $\vec{B}$ is the magnetic flux density or magnetic field. Eq. (1) implies that $\vec{H}$ is a conservative vector field and therefore it can be expressed as the gradient of a scalar magnetic potential $V_{m}$.

Furthermore, $\vec{B}$ depends on the material in which fields are present through the constitutive equation:

$$
\vec{B}=\mu_{0}(\vec{H}+\vec{M})
$$

where $\mu_{0}$ is the magnetic permeability of vacuum and $\vec{M}$ is the magnetization. In linear materials, $\vec{M}$ can be obtained from Eq. (4):

$$
\vec{M}=X \cdot \vec{H}
$$

where $X$ represents the magnetic susceptibility. For $0.9 \% \mathrm{NaCl}$ samples, $X$ was calculated according to the Wiedemann's additivity law: 
where $V_{\text {water }}$ and $V_{\mathrm{NaCl}}$ are the volume of pure water and $\mathrm{NaCl}$ in the solution, while $X_{\text {water }}$ and $X_{\mathrm{Nacl}}$ are the magnetic susceptibility of pure water and $\mathrm{NaCl}$, respectively (Lide, 2003-2004).

In magnets, which are non-linear, $\vec{B}$ was expressed as the sum of a proportional term and the magnet remanence $\overrightarrow{B_{r}}$ :

$$
\vec{B}=\mu \vec{H}+\overrightarrow{B_{r}}
$$

where $\mu=1.05 \cdot \mu_{0}$ is the permeability of neodymium.

Moreover, two boundary conditions were considered for the resolution of the problem:

$$
\begin{gathered}
\vec{n} \cdot \vec{B}=0 \\
V_{m}=0
\end{gathered}
$$

Eq. (7) assumes that the SMF lines do not cut any of the infinite planes which contain both magnet axes, while Eq. (8) refers to the middle plane between both magnets, where the SMF lines are perpendicular, involving $V_{m}$ constant, which has been taken zero.

Different computational grids were used for the numerical solution of the problem in order to provide a mesh independent solution. After solving Eqs. (1) - (8), the SMF strength and the direction of the field lines were obtained in the domain considered to characterize accurately the SMFs applied in the sample during freezing.

To corroborate the simulated results, the magnetic field strength was measured, using a teslameter (model GM07 equipped with a thin semi-flexible transverse Hall probe TP002, Hirst Magnetic Instruments LTD, Falmouth, UK) with an accuracy better than $\pm 1 \%$, at seven different positions between the magnets arranged both in attractive and repulsive position (Fig. 1).

\subsection{Analysis of the freezing curves}

Freezing curves were analyzed to obtain some characteristic parameters of the freezing process: the time at which nucleation occurred, the temperature at the sample center when nucleation was triggered, the extent of supercooling attained at the sample center, and the phase transition and total freezing times (Fig. 2a).

The time at which nucleation occurred, $t_{\text {nuc }}(\mathrm{s})$, was recognized in the freezing curves as the time at which a sudden temperature increase took place at the vial surface due to the release of latent heat from the sample. At that moment, $T_{c}^{\text {nuc }}\left({ }^{\circ} \mathrm{C}\right)$ was the temperature at the sample center. When $T_{c}^{\text {nuc }}$ was lower than the freezing point of the sample $\left(T_{f p}=0^{\circ} \mathrm{C}\right.$ for pure water and $T_{f p}=-0.6{ }^{\circ} \mathrm{C}$ for $0.9 \% \mathrm{NaCl}$ solutions), the extent of supercooling attained at the sample center, $\Delta T_{c}\left({ }^{\circ} \mathrm{C}\right)$, was calculated as the difference between $T_{f p}$ and $T_{c}^{\text {nuc }}$. In other cases, no supercooling existed at the sample center and $\Delta T_{c}$ was considered to be zero. 
The phase transition time, $t_{p t}(\mathrm{~s})$, was defined in this paper as the time span between nucleation and the end point of freezing. The end point of freezing was identified from the slope of the freezing curve recorded at the sample center (Rahman et al., 2002). To do so, the first derivative of the freezing curve was obtained by using the software Matlab (v. 7.11.0.584 (R2010b), MathWorks Inc., Natick, MA, USA) and analyzed (Fig. 2b). During the freezing plateau, the slope is zero because temperature remains constant at the initial freezing point due to the release of latent heat. When ice formation starts to decrease, the slope starts to increase up to a maximum that indicates the phase change is completed (Rahman et al., 2002). In this paper, this maximum is considered to be the end point of freezing.

The total freezing time, $t_{\text {tot }}(\mathrm{s})$, was the time required to lower the sample temperature from $25^{\circ} \mathrm{C}$ (initial sample temperature) to $-20^{\circ} \mathrm{C}$.

229

\subsection{Statistical analysis}

The statistical analysis of the characteristic parameters recorded in the freezing experiments $\left(t_{n u c}, T_{c}^{n u c}, \Delta T_{c}, t_{p t}\right.$, and $t_{\text {tot }}$ ) was performed using the software program IBM SPSS Statistics v. 23.0.0.0 for Windows (IBM Corp., Armonk, NY, USA). The Shapiro-Wilk and the Levene tests were employed to check the normality and homoscedasticity of the data, respectively. In those cases in which the data conformed a normal distribution, a one-way analysis of variance (ANOVA) was performed to detect whether the means of the characteristic parameters registered in control, SMF-A, and SMF-R freezing experiments were all equal or not. When the assumption of normality was not confirmed, the non-parametric Kruskal-Wallis test was employed to compare the characteristic parameters of the different freezing experiments. The significance level was set at $5 \%$.

\section{RESULTS AND DISCUSSION}

\subsection{Characterization of the static magnetic fields produced during the experiments}

The static magnetic fields produced by the magnets during the SMF-A and SMF-R freezing experiments were simulated by solving the mathematical model described in section 2.3. Fig. 3 clearly shows that the relative position of the magnet poles in the experimental device affected the magnetic field direction, strength, and the spatial magnetic gradients established throughout the sample.

Figs. $3 \mathrm{a}$ and $3 \mathrm{~b}$ depict the magnetic field direction and strength in the complete computational domain when the magnets were arranged in attractive and repulsive position, respectively. The magnetic field strength decreased when increasing the distance to the magnets as expected. For each magnet, magnetic field lines spread out from the north pole, curve around the magnet, 
and return to the south pole. Moreover, when the magnets were arranged in attractive position, the field lines between both magnets ran directly from one magnet to the other.

Figs. $3 c$ and $3 d$ reveal that substantial spatial magnetic gradients were established in the water samples during the SMF-A and SMF-R experiments. In both cases, the magnetic field strength reached its maximum intensity at the sample surface closest to the magnets (359 mT and 241 $\mathrm{mT}$ in the attractive and repulsive arrangements, respectively). These maximum intensities were about 4 orders of magnitude larger than that of the Earth's natural magnetic field (0.045 mT in Madrid according to the National Center for Environmental Information (n. d.)). Then, magnetic field strength progressively declined towards the sample center and, thus, minimum $\vec{B}$ values were found at the center of the top and bottom edges of the sample in SMF-A experiments (107 $\mathrm{mT}$ ) and at its geometric center in SMF-R experiments (close to $0 \mathrm{mT}$ ). Therefore, the arrangement with unlike magnet poles faced each other produced a stronger magnetic field in the sample as expected. When like magnet poles were faced each other, the SMF strength was significantly weaker and it vanished at the geometric center of the sample. Therefore, this configuration seems to be less appropriate to evaluate the effects of SMFs.

To corroborate the results obtained from the mathematical model, the magnetic field strength was measured, by using a teslameter, at seven points between the two magnets arranged both in attractive and repulsive position. Fig. 4 shows that the maximum difference found between the experimental and the modeled data was $30 \mathrm{mT}$. Taking into account the inherent inaccuracy on situating the probe at an exact position during the measurements, the experimental data agreed well with the results obtained by the mathematical model.

The SMFs established between the magnets when freezing $0.9 \% \mathrm{NaCl}$ solutions were very similar to those calculated for pure water samples. The magnetic susceptibility of $0.9 \% \mathrm{NaCl}$ has a value very close to that of water; namely $X_{\text {water }}=-9.046 \cdot 10^{-6}$ and $X_{0.9 \% \mathrm{NaCl}}=-9.067 \cdot 10^{-6}$. Therefore, no significant changes were observed in the strength and orientation of the magnetic field vectors when freezing pure water or $\mathrm{NaCl}$ solutions (data not shown).

\subsection{Effect of static magnetic fields on water freezing}

282 Typical time-temperature plots obtained during conventional freezing experiments of pure water are shown in Figs. $5 \mathrm{a}$ and $5 \mathrm{~b}$. During the freezing process, thermal gradients were established along the samples. Thus, the temperature at the glass-vial surface, a rough indicator of the temperature at the sample surface (vial-wall thickness $<1 \mathrm{~mm}$ ), was always lower than that the sample center. The static magnetic fields applied in this paper did not affect the shape or the appearance of the freezing curves and the time-temperature plots obtained in SMF experiments were similar to those depicted in Fig. 5 (plots not shown).

The freezing curves clearly exhibited the three key steps of the process: precooling, phase transition, and tempering. In the precooling step, the cooling of the sample implied the removal 
of only sensible heat. Once the freezing point of pure water $\left(T_{f p}=0^{\circ} \mathrm{C}\right)$ was reached at the sample surface, ice nucleation did not occur immediately in any case, but all the samples supercooled to a temperature well below $T_{f p}$. Then, after reaching a certain extent of supercooling, ice nucleation suddenly occurred.

Fig. 6a certainly shows the stochastic nature of ice nucleation. Thus, and according to the literature (Heneghan et al., 2002; Reid, 1983), we found that ice nucleation did not occur at the same time or after reaching the same extent of supercooling in repeated experiments. At the conditions tested in this paper, ice nucleation was triggered between $67 \mathrm{~s}$ and $175 \mathrm{~s}$ after immersing the sample in the cooling medium when the temperature at the sample center ranged between $10.4^{\circ} \mathrm{C}$ and $-11.1^{\circ} \mathrm{C}$.

Fig. 6a revealed no effect of the SMF application on both $t_{\text {nuc }}$ and $T_{c}^{\text {nuc }}\left(p>0.05\right.$, Table 1). Due to the thermal gradients established, $T_{c}^{\text {nuc }}$ is the temperature at the hottest point in the sample when nucleation occurred and, therefore, $\Delta T_{c}$ represents the minimum supercooling reached throughout the sample. Obviously, the later the nucleation occurred, the lower $T_{c}^{n u c}$, the larger the extent of supercooling reached throughout the sample and, consequently, the larger $\Delta T_{c}$ (Fig. 6a). For example, in Fig. 5a, ice nucleation was triggered early, namely $75 \mathrm{~s}$ after the onset of the freezing experiment. At this moment, the sample surface was supercooled $\left(\Delta T_{s} \sim 10.6^{\circ} \mathrm{C}\right)$, but the temperature at the sample center was still above $T_{f p}\left(T_{c}^{n u c}=10.1^{\circ} \mathrm{C}\right)$, that means, $\Delta T_{c}=0$. Therefore, ice nuclei were formed only at the sample surface where enough extent of supercooling had been reached. By contrast, in Fig. 5b, ice nucleation was triggered much later, namely, $132 \mathrm{~s}$ after immersing the sample in the cooling medium. At this time, the sample was completely supercooled $\left(\Delta T_{s} \sim 19.5^{\circ} \mathrm{C}\right.$ and $\Delta T_{c}=$ $6.6^{\circ} \mathrm{C}$ ) and, therefore, ice nucleation took place throughout the whole sample and not only at the surface. When no SMFs were applied, complete supercooling of the whole sample before nucleation or, in other words, $\Delta T_{c}>0{ }^{\circ} \mathrm{C}$, occurred in 14 of 30 experiments. This proportion was similar to that observed when the magnets were arranged either in attractive or in repulsive position (16 of 30 experiments and 18 of 30 experiments, respectively). Moreover, in these experiments in which supercooling occurred at the sample center $\left(\Delta T_{c}>0\right), \Delta T_{c}$ was not significantly affected by the SMF application ( $p>0.05$, Table 1 ) and, thus, mean $\Delta T_{c}$ values were close to $5{ }^{\circ} \mathrm{C}$ in all cases (Table 2). Therefore, in contrast to some results reported in the literature (Aleksandrov et al., 2000; Zhou et al., 2012) and according to Zhao et al. (2017), we did not find any effect of SMFs on water supercooling.

323 After nucleation, crystal growth occurs by the addition of water molecules to the nuclei formed. 324 During the phase transition step, the temperature at the center of the sample remained constant at $T_{f p}$ until all the water was converted to ice and the latent heat of crystallization was removed

326 (Figs. 5a and 5b). Fig. 7a confirms previous data in the literature (Le Bail et al., 1997; Otero and 327 Sanz, 2000, 2006) that show that the larger the extent of supercooling attained throughout the 328 sample (or, in other words, the longer the nucleation time), the larger the amount of ice instantaneously formed at nucleation and, therefore, the shorter the phase transition step. In 
this paper, the phase transition time ranged between $381 \mathrm{~s}$ and $462 \mathrm{~s}$ in repeated experiments and no effect of SMFs was detected ( $p>0.05$, Table 1$)$. Once all water was transformed into ice, the sample temperature decreased while sensible heat was removed during the tempering step (Figs. 5a and 5b). We did not observe any effect of SMFs on the rate of heat removal during the freezing process and, thus, the total freezing times did not differ significantly in control, SMF-A, and SMF-R experiments ( $p>0.05$, Table 1$)$.

\subsubsection{Effect of static magnetic fields on freezing of $0.9 \% \mathrm{NaCl}$ solutions}

The time-temperature plots obtained during control experiments in $0.9 \% \mathrm{NaCl}$ solutions (Figs. $5 c$ and $5 d$ ) were similar in shape and appearance to those recorded for pure water except in the temperature at the freezing plateau $\left(T_{f p}=-0.6^{\circ} \mathrm{C}\right)$. When static magnetic fields were applied, the freezing curves were not visually affected and the SMF plots seem to be identical to the control ones (plots not shown).

As occurred in pure water, we did not detect any effect of the SMFs applied, whichever the direction of the field forces, on supercooling. In $0.9 \% \mathrm{NaCl}$ solutions, ice nucleation occurred between $77 \mathrm{~s}$ and $154 \mathrm{~s}$ after immersing the sample in the cooling medium (Fig. 6b) and $t_{\text {nuc }}$ distributions were similar in control, SMF-A, and SMF-R experiments $(p>0.05$, Table 1$)$. Depending on the nucleation time, the samples were supercooled in a greater or lesser extent (Figs. $5 \mathrm{c}$ and $5 \mathrm{~d}$ ) and, thus, $T_{c}^{\text {nuc }}$ ranged between $8.3^{\circ} \mathrm{C}$ and $-9.8^{\circ} \mathrm{C}$ (Fig. 6b). When no SMFs were applied, complete supercooling of the entire sample, that is, $\Delta T_{c}>0$, occurred in 18 of 30 experiments. Similar proportions, $15 / 30$ and 17/30, were observed in SMF-A and SMF-R experiments, respectively. $\Delta T_{c}$, when existed, ranged between $0.5^{\circ} \mathrm{C}$ to $9.2^{\circ} \mathrm{C}$ and no effect of SMFs on either $T_{c}^{\text {nuc }}$ or $\Delta T_{c}$ was detected $(p>0.05$, Table 1$)$.

The phase transition and total freezing times were similar in control, SMF-A, and SMF-R experiments (Fig. 7b, Table 2). Thus, in contrast to the results reported by Mok et al. (2015) and Zhao et al. (2017), we did not find any effect of SMFs, whichever the direction of the field forces, on the freezing kinetics of $0.9 \% \mathrm{NaCl}$ solutions ( $p>0.05$, Table 1$)$.

\section{CONCLUSIONS}

During SMF experiments, significant spatial magnetic gradients were established throughout the samples despite their relatively small size and their location close to the magnets. Thus, $\vec{B}$ values ranged from 107 to $359 \mathrm{mT}$ and from 0 to $241 \mathrm{mT}$ in different points of the sample in SMF-A and SMF-R experiments, respectively. At these conditions, we did not find any SMF effect, whichever the field orientation, on either supercooling or freezing kinetics of both pure water samples and $0.9 \% \mathrm{NaCl}$ solutions. 
Our results make it clear that an accurate characterization of the static magnetic fields actually applied in the entire volume of the sample is essential to assess the SMF effects on freezing. Otherwise, the real SMFs applied could be over- or underestimated, hypothetical SMF effects could be masked by large spatial magnetic gradients, incorrect conclusions could be drawn, and comparisons among different laboratories would be impossible.

Future research works could be focused on evaluating the effect of more uniform static magnetic fields, in a wider $\vec{B}$ range and in smaller samples, to better elucidate any SMF effect on supercooling and freezing kinetics. In any case, it is important to note that, when freezing real foods, very much larger spatial magnetic gradients than those observed in this paper should be expected and this could hamper the implementation of this technology in the food industry.

\section{Acknowledgments}

This work was supported by the Spanish Ministry of Economy and Competitiveness (MINECO) through the project AGL2012-39756-C02-01. Antonio C. Rodríguez acknowledges the predoctoral contract BES-2013-065942 from MINECO, jointly financed by the European Social Fund, in the framework of the National Program for the Promotion of Talent and its Employability (National Sub-Program for Doctors Training). The authors thank Javier SánchezBenítez, researcher at Universidad Complutense de Madrid (Facultad de Químicas), for his help in the design and construction of the device for holding the sample and the magnets.

\section{REFERENCES}

Aleksandrov, V., Barannikov, A., Dobritsa, N., (2000). Effect of magnetic field on the supercooling of water drops. Inorganic Materials 36(9), 895-898.

Beaugnon, E., Tournier, R., (1991). Levitation of water and organic substances in high static magnetic fields. Journal de Physique III, EDP Sciences 1(8), 1423-1428.

Cai, R., Yang, H., He, J., Zhu, W., (2009). The effects of magnetic fields on water molecular hydrogen bonds. Journal of Molecular Structure 938(1-3), 15-19.

Chang, K.T., Weng, C.I., (2006). The effect of an external magnetic field on the structure of liquid water using molecular dynamics simulation. Journal of Applied Physics 100(4), 043917043916.

Chen, Z., Dahlberg, E.D., (2011). Deformation of water by a magnetic field. The Physics Teacher 49(3), 144-146.

Erikson, U., Kjørsvik, E., Bardal, T., Digre, H., Schei, M., Søreide, T.S., Aursand, I.G., (2016). Quality of Atlantic cod frozen in cell alive system, air-blast, and cold storage freezers. Journal of Aquatic Food Product Technology, 1-20.

Heneghan, A.F., Wilson, P.W., Haymet, A.D.J., (2002). Heterogeneous nucleation of supercooled water, and the effect of an added catalyst. Proceedings of the National Academy of Sciences of the United States of America 99(15), 9631-9634.

Hosoda, H., Mori, H., Sogoshi, N., Nagasawa, A., Nakabayashi, S., (2004). Refractive indices of water and aqueous electrolyte solutions under high magnetic fields. The Journal of Physical Chemistry A 108(9), 1461-1464.

Ikezoe, Y., Hirota, N., Nakagawa, J., Kitazawa, K., (1998). Making water levitate. Nature 393(6687), 749-750. 
Inaba, H., Saitou, T., Tozaki, K.I., Hayashi, H., (2004). Effect of the magnetic field on the melting 410 transition of $\mathrm{H}_{2} \mathrm{O}$ and $\mathrm{D}_{2} \mathrm{O}$ measured by a high resolution and supersensitive differential 411 scanning calorimeter. Journal of Applied Physics 96(11), 6127-6132.

412 James, C., Reitz, B., James, S.J., (2015). The freezing characteristics of garlic bulbs (Allium 413 sativum L.) frozen conventionally or with the assistance of an oscillating weak magnetic field. 414 Food and Bioprocess Technology 8(3), 702-708.

415 Le Bail, A., Chourot, J.M., Barillot, P., Lebas, J.M., (1997). Congélation-decongélation a haute 416 pression. Revue Generale du Froid 972, 51-56.

417 Lide, D.R., (2003-2004). CRC Handbook of chemistry and physics: A ready-reference book of 418 chemical and physical data $\left(84^{\text {th }}\right.$ ed). CRC Press, Boca Raton, FL.

419 Lou, Y.-j., Zhao, H.-x., Li, W.-b., Han, J.-t., (2013). Experimental of the effects of static magnetic Mok, J.H., Choi, W., Park, S.H., Lee, S.H., Jun, S., (2015). Emerging pulsed electric field (PEF) and static magnetic field (SMF) combination technology for food freezing. International Journal of Refrigeration 50, 137-145.

National Centers for Environmental Information, (n.d.). Magnetic field calculators. Retrieved from https://www.ngdc.noaa.gov/geomag-web/\#igrfwmm. Last accesssed date: 2017 Augost 4.

Otero, L., Pérez-Mateos, M., Rodríguez, A.C., Sanz, P.D., (2017). Electromagnetic freezing: Effects of weak oscillating magnetic fields on crab sticks. Journal of Food Engineering 200, 8794.

Otero, L., Rodríguez, A.C., Pérez-Mateos, M., Sanz, P.D., (2016). Effects of magnetic fields on freezing: Application to biological products. Comprehensive Reviews in Food Science and Food Safety 15(3), 646-667.

Otero, L., Sanz, P.D., (2000). High-pressure shift freezing. Part 1. Amount of ice instantaneously formed in the process. Biotechnology Progress 16(6), 1030-1036.

Otero, L., Sanz, P.D., (2006). High-pressure-shift freezing: Main factors implied in the phase transition time. Journal of Food Engineering 72(4), 354-363.

Owada, N., (2007). Highly-efficient freezing apparatus and high-efficient freezing method. US Patent 7237400 B2.

Owada, N., Saito, S., (2010). Quick freezing apparatus and quick freezing method. US Patent 7810340 B2.

Pang, X.F., Deng, B., Tang, B., (2012). Influences of magnetic field on macroscopic properties of water. Modern Physics Letters B 26(11), 1250069.

Pang, X., Deng, B., (2008a). The changes of macroscopic features and microscopic structures of water under influence of magnetic field. Physica B: Condensed Matter 403(19-20), 3571-3577.

Pang, X., Deng, B., (2008b). Investigation of changes in properties of water under the action of a magnetic field. Science in China Series G: Physics, Mechanics and Astronomy 51(11), 16211632.

Petzold, G., Aguilera, J.M., (2009). Ice morphology: Fundamentals and technological applications in foods. Food Biophysics 4(4), 378-396.

Rahman, M.S., Guizani, N., Al-Khaseibi, M., Ali Al-Hinai, S., Al-Maskri, S.S., Al-Hamhami, K., (2002). Analysis of cooling curve to determine the end point of freezing. Food Hydrocolloids 16(6), 653-659.

Reid, D.S., (1983). Fundamental physicochemical aspects of freezing. Food Technology 37(4), 110-115.

Sato, M., Fujita, K., (2008). Freezer, freezing method and frozen objects. US Patent 7418823 B2.

Szcześ, A., Chibowski, E., Hołysz, L., Rafalski, P., (2011). Effects of static magnetic field on water at kinetic condition. Chemical Engineering and Processing: Process Intensification 50(1), 124127. 
Senesi, R., Flammini, D., Kolesnikov, A. I., Murray, E. D., Galli, G., Andreani, C., (2013). The quantum nature of the $\mathrm{OH}$ stretching mode in ice and water probed by neutron scattering experiments. The Journal of Chemical Physics 139 (7), 074504. Toledo, E.J.L., Ramalho, T.C., Magriotis, Z.M., (2008). Influence of magnetic field on physicalchemical properties of the liquid water: Insights from experimental and theoretical models. Journal of Molecular Structure 888(1-3), 409-415.

466 Wang, Y., Zhang, B., Gong, Z., Gao, K., Ou, Y., Zhang, J., (2013). The effect of a static magnetic

467 field on the hydrogen bonding in water using frictional experiments. Journal of Molecular 468 Structure 1052(0), 102-104.

469 Zaritzky, N., (2011). Physical-chemical principles in freezing. In: Sun, D.W. (Ed.), Handbook of Frozen Food Processing and Packaging, Second Edition. CRC Press, Boca Raton, pp. 3-37.

471 Zhao, H., Hu, H., Liu, S., Han, J., (2017). Experimental study on freezing of liquids under static magnetic field. Biotechnology and Bioengineering.

473 Zhou, K.X., Lu, G.W., Zhou, Q.C., Song, J.H., Jiang, S.T., Xia, H.R., (2000). Monte Carlo simulation 474 of liquid water in a magnetic field. Journal of Applied Physics 88(4), 1802-1805.

475 Zhou, Z., Zhao, H., Han, J., (2012). Supercooling and crystallization of water under DC magnetic 476 fields. CIESC Journal 63(5), 1405-1408. 
$479 \mathrm{p}$-values obtained after applying the Shapiro-Wilk test to check the normality of the data and the 480 Kruskal-Wallis and ANOVA tests to compare the characteristic parameters of control (no SMF 481 application), SMF-A, and SMF-R freezing experiments. $t_{\text {nuc }}$ : Time at which nucleation occurred 482 (s), $T_{c}^{\text {nuc }}$ : Temperature at the sample center when nucleation occurred $\left({ }^{\circ} \mathrm{C}\right), \Delta T_{c}$ : Extent of 483 supercooling at the sample center $\left({ }^{\circ} \mathrm{C}\right)$ if exists $\left(\Delta T_{c}>0\right), t_{p t}$ : Phase transition time (s), and $t_{\text {tot: }}$ : 484 Total freezing time (s).

485

\begin{tabular}{|c|c|c|c|c|c|}
\hline & \multicolumn{3}{|c|}{ Shapiro-Wilk } & \multirow{2}{*}{ ANOVA } & \multirow{2}{*}{$\begin{array}{l}\text { Kruskal- } \\
\text { Wallis }\end{array}$} \\
\hline & No SMF & SMF-A & SMF-R & & \\
\hline \multicolumn{6}{|c|}{ Pure water samples } \\
\hline$t_{n u c}$ & 0.003 & 0.002 & 0.014 & -- & 0.408 \\
\hline$T_{c}^{\text {nuc }}$ & 0.000 & 0.000 & 0.004 & -- & 0.440 \\
\hline$\Delta T_{c}$ & 0.191 & 0.063 & 0.170 & 0.996 & -- \\
\hline$t_{p t}$ & 0.001 & 0.015 & 0.113 & -- & 0.619 \\
\hline$t_{\text {tot }}$ & 0.079 & 0.126 & 0.097 & 0.068 & -- \\
\hline \multicolumn{6}{|c|}{$0.9 \% \mathrm{NaCl}$ solutions } \\
\hline$t_{\text {nuc }}$ & 0.005 & 0.067 & 0.415 & -- & 0.830 \\
\hline$T_{c}^{\text {nuc }}$ & 0.000 & 0.001 & 0.008 & -- & 0.742 \\
\hline$\Delta T_{c}$ & 0.073 & 0.611 & 0.730 & 0.577 & -- \\
\hline$t_{p t}$ & 0.027 & 0.042 & 0.022 & -- & 0.827 \\
\hline$t_{\text {tot }}$ & 0.917 & 0.576 & 0.814 & 0.837 & -- \\
\hline
\end{tabular}

486

487

488 
490 Mean \pm standard error values of the characteristic parameters of control (no SMF application), 491 SMF-A, and SMF-R freezing experiments. $t_{\text {nuc }}$ : Time at which nucleation occurred $(\mathrm{s}), T_{c}^{\text {nuc }}$ : 492 Temperature at the sample center when nucleation occurred $\left({ }^{\circ} \mathrm{C}\right), \Delta T_{c}$ : Extent of supercooling 493 at the sample center $\left({ }^{\circ} \mathrm{C}\right)$ if exists $\left(\Delta T_{c}>0\right), t_{p t}$ : Phase transition time (s), and $t_{\text {tot }}$ : Total freezing 494 time (s).

495

\begin{tabular}{lccc}
\hline & No SMF & SMF-A & SMF-R \\
\hline Pure water samples & & & \\
\cline { 1 - 1 }$t_{\text {nuc }}$ & $99 \pm 4$ & $103 \pm 4$ & $106 \pm 5$ \\
$T_{c}^{\text {nuc }}$ & $2.6 \pm 1.3$ & $1.5 \pm 1.3$ & $0.5 \pm 1.3$ \\
$\Delta T_{c}$ & $4.8 \pm 0.7$ & $4.8 \pm 0.6$ & $4.8 \pm 0.7$ \\
$t_{p t}$ & $430 \pm 4$ & $430 \pm 4$ & $425 \pm 4$ \\
$t_{\text {tot }}$ & $605 \pm 2$ & $611 \pm 2$ & $605 \pm 2$ \\
\hline $\mathbf{0 . 9 \% ~ N a C l ~ s o l u t i o n s ~}$ & $115 \pm 4$ & $110 \pm 4$ & $110 \pm 3$ \\
\hline$t_{\text {nuc }}$ & $-0.9 \pm 1.2$ & $0.2 \pm 1.2$ & $-0.5 \pm 1.0$ \\
$T_{c}^{\text {nuc }}$ & $5.6 \pm 0.5$ & $5.4 \pm 0.7$ & $4.3 \pm 0.5$ \\
$\Delta T_{c}$ & $437 \pm 3$ & $440 \pm 4$ & $440 \pm 3$ \\
$t_{\text {pt }}$ & $637 \pm 3$ & $635 \pm 2$ & $636 \pm 2$ \\
$t_{\text {tot }}$ & & &
\end{tabular}
496

497

498 
Schematic draw of the device fabricated for holding the sample and the magnets during the SMF freezing experiments. (1): PMMA block, (2) Neodymium magnet, (3) Removable PMMA lid, (4): Teflon ${ }^{\circledR}$ bolt, (5): Teflon ${ }^{\circledR}$ nut, and (6): Sample vial. (a-g): Positions at which the magnetic field strength was experimentally measured.

506

(a) Characteristic parameters of the freezing process ( $t_{\text {nuc }}$ : Nucleation time, $T_{c}^{\text {nuc }}$ : Temperature at the sample center when nucleation occurred, $\Delta T_{c}$ : Extent of supercooling at the sample center, $t_{p t}$ : Phase transition time, and $t_{\text {tot: }}$ Total freezing time) obtained from the freezing curves. (-): Temperature at the sample center. (---): Temperature at the vial surface. (b): Slope of the freezing curve at the sample center. Magnetic field direction and strength $(\mathrm{mT})$ calculated by solving the mathematical model described in section 2.3. (a-b): Complete computational domain when the magnets were arranged in either attractive or repulsive position, respectively. (c-d): Detail of the water sample when the magnets were arranged in either attractive or repulsive position, respectively.

X-component of the magnetic field strength at the points defined in Fig. 1. X: Experimental measurements. $\circ$ : Modeled data.

Temperature evolution at the sample center (-) and the vial surface (---) during freezing experiments in (a-b): pure water and (c-d): $0.9 \% \mathrm{NaCl}$ solutions with no SMF application. (a and $c$ ): Typical experiments with partial supercooling of the sample $\left(\Delta T_{c}=0{ }^{\circ} \mathrm{C}\right)$ and $(\mathrm{b}$ and $\mathrm{d})$ : Typical experiments with complete supercooling of the whole sample $\left(\Delta T_{c}>0{ }^{\circ} \mathrm{C}\right) . \Delta T_{c}$ : Extent of supercooling reached at the sample center just before nucleation. Key steps of the process: Temperature $\left({ }^{\circ} \mathrm{C}\right)$ and extent of supercooling $\left({ }^{\circ} \mathrm{C}\right)$ at the sample center when nucleation occurred in $(+)$ : control, $(\odot)$ : SMF-A, and $(\Delta)$ : SMF-R experiments. a) Pure water samples and b) $0.9 \% \mathrm{NaCl}$ solutions. 
Phase transition time (s) in $(+)$ : control, $(0)$ : SMF-A, and $(\Delta)$ : SMF-R experiments. a) Pure water samples and b) $0.9 \% \mathrm{NaCl}$ solutions.

537

538 
539 FIGURE 1

540

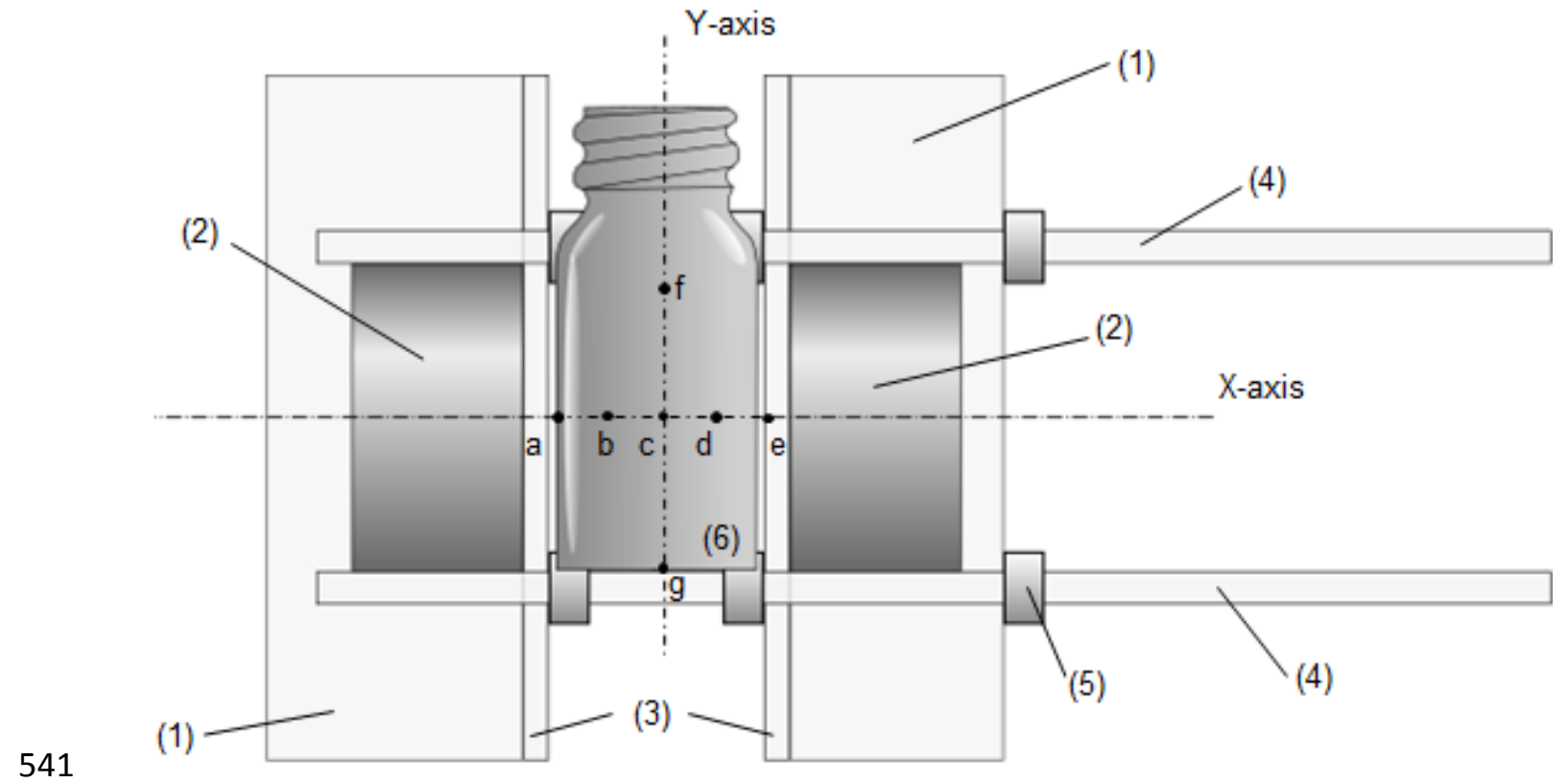

542 
a)

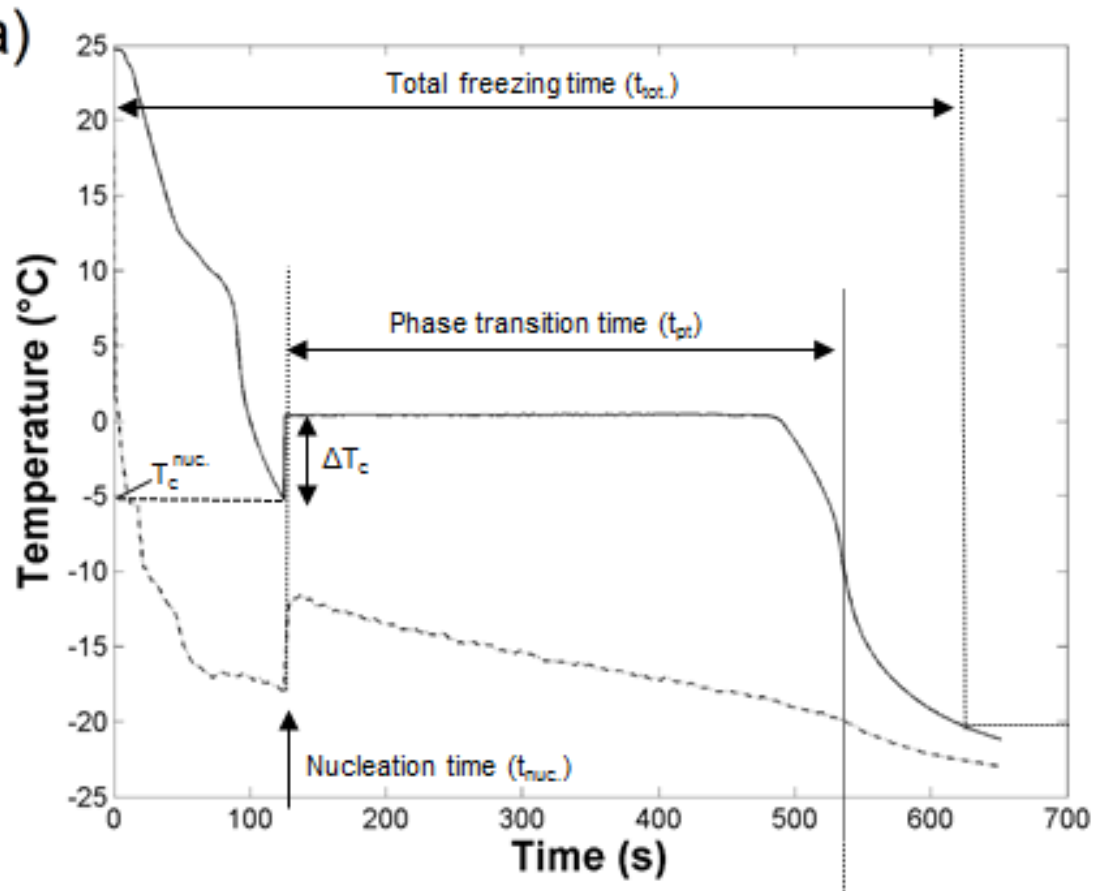

b)

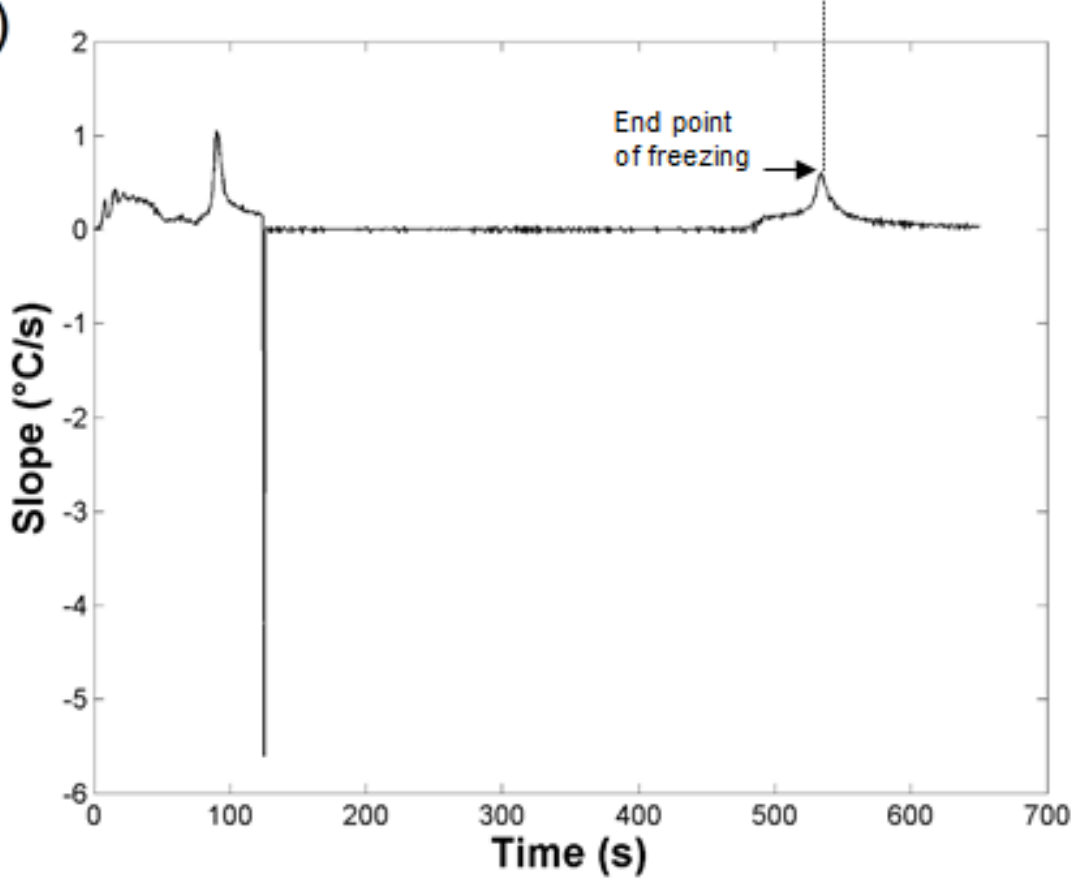


(a)

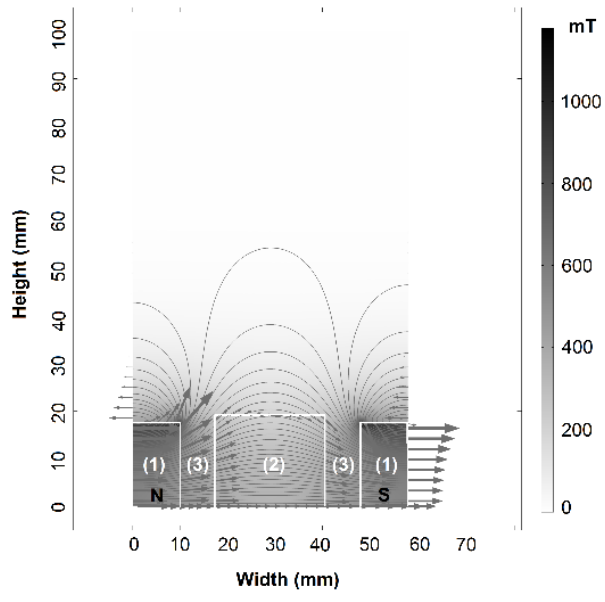

c)

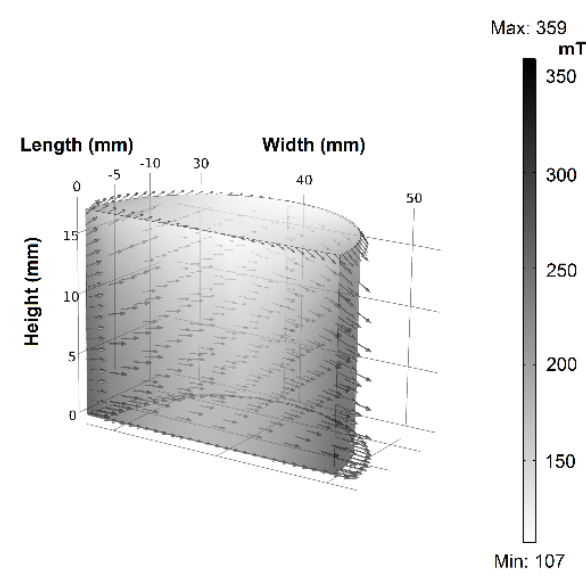

b)

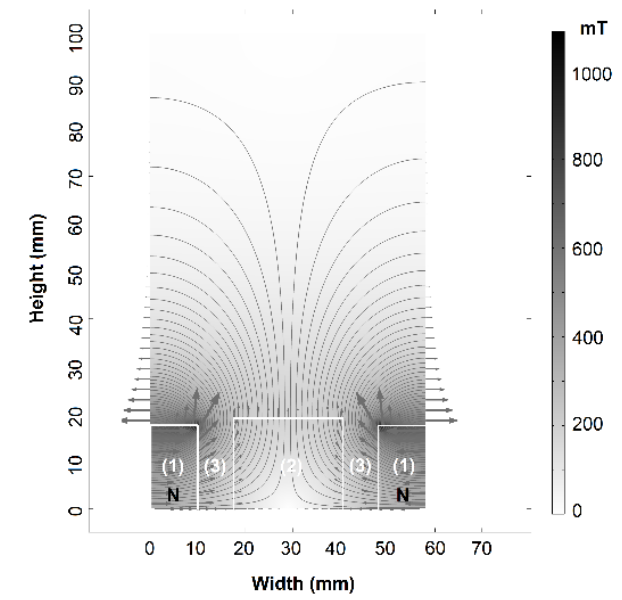

d)

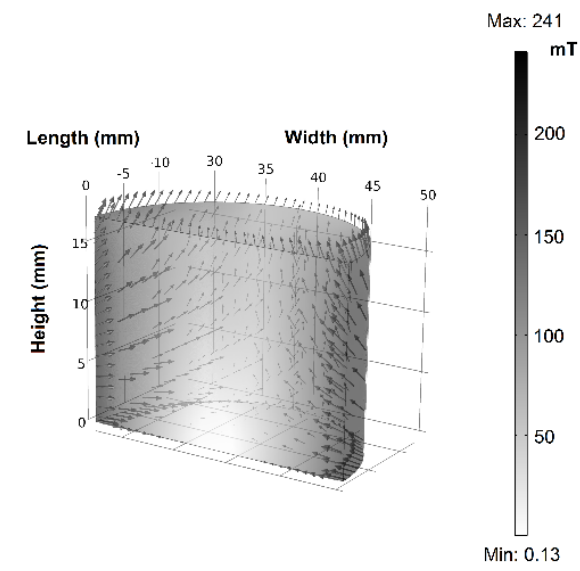


552

a)

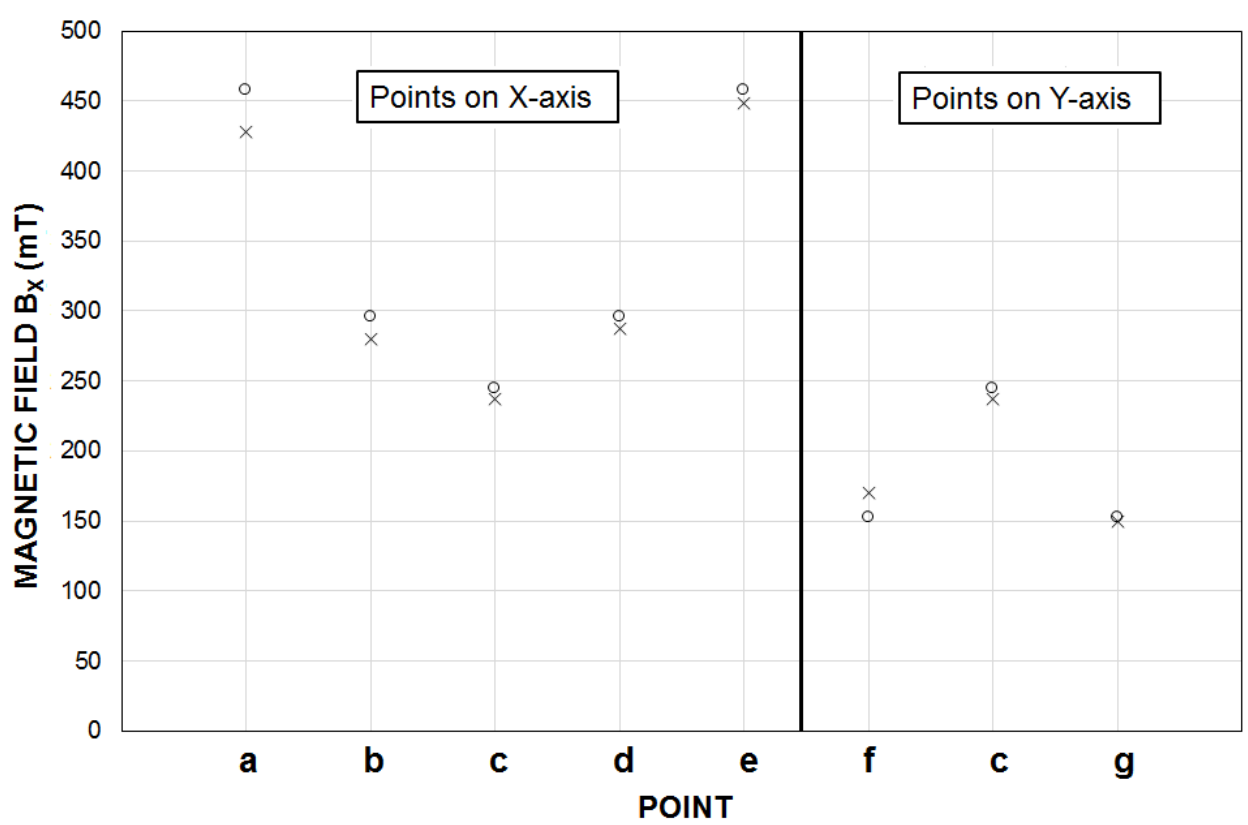

b)

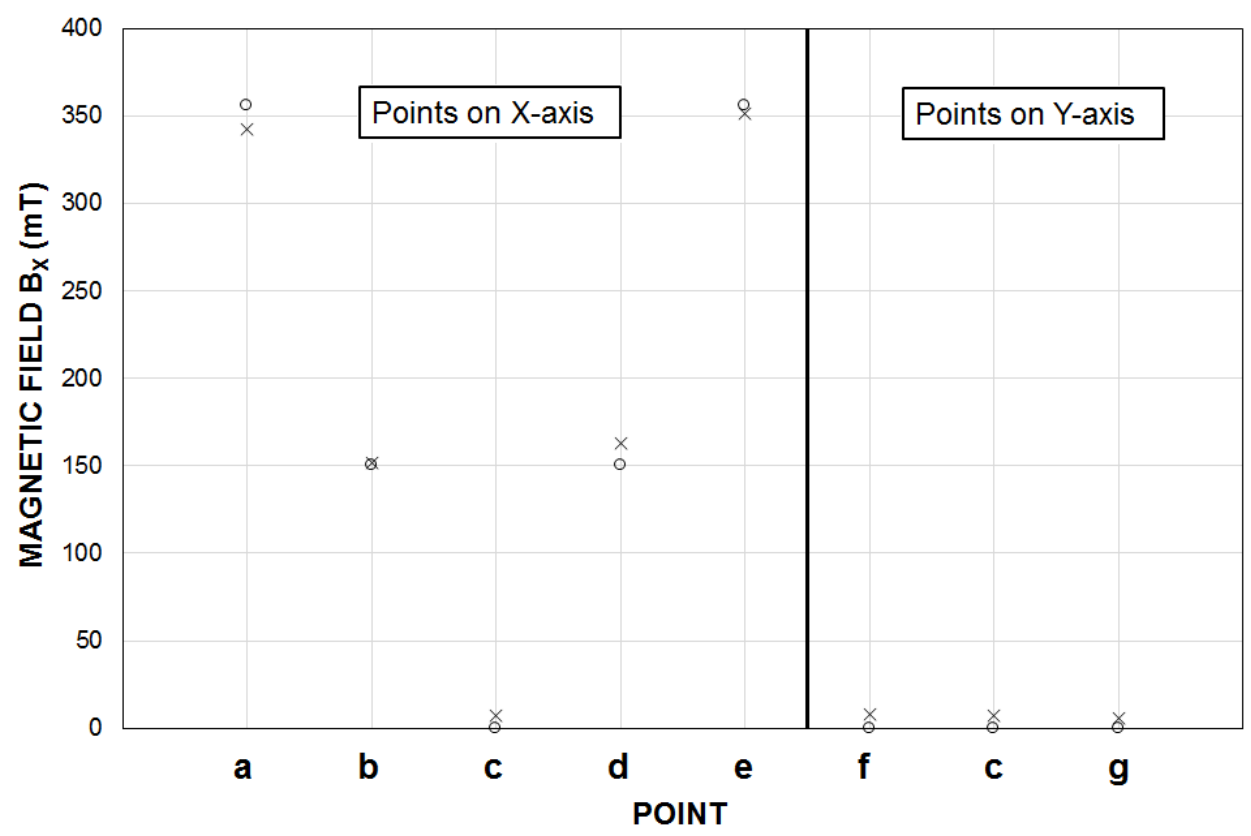

553 
FIGURE 5

556

a)

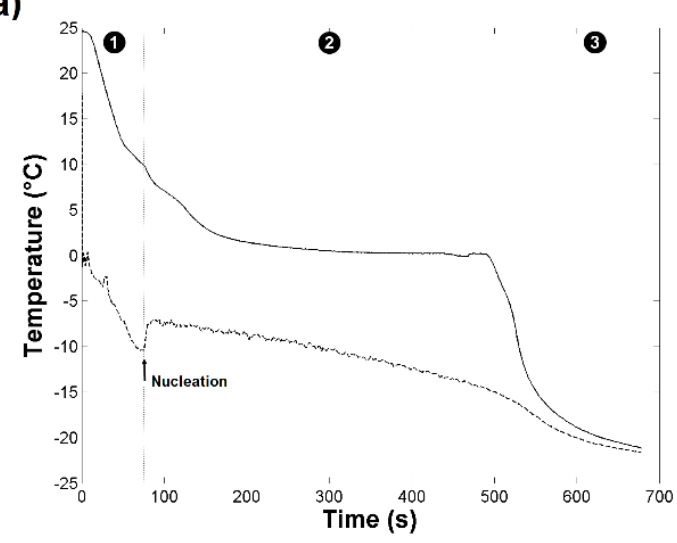

c)

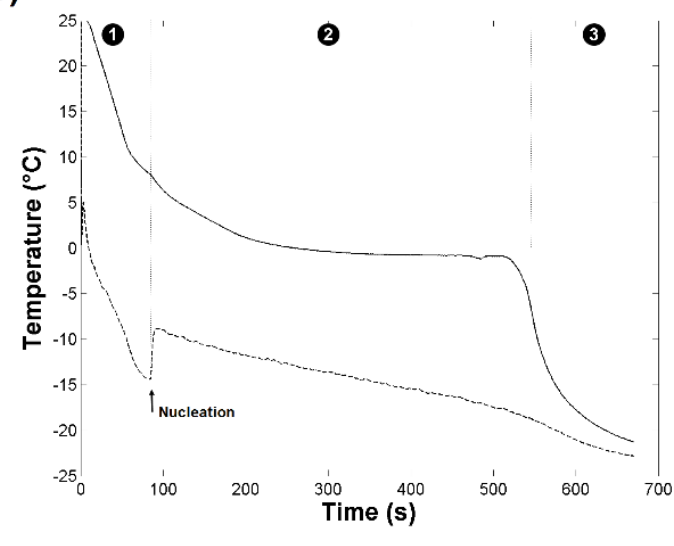

b)

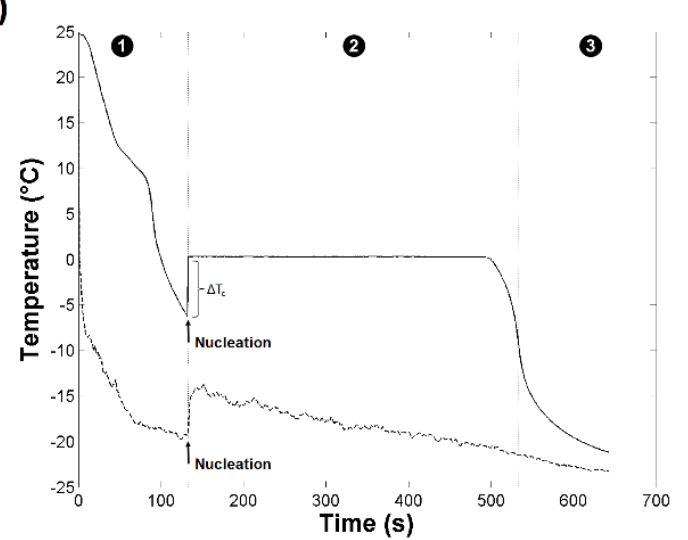

d)

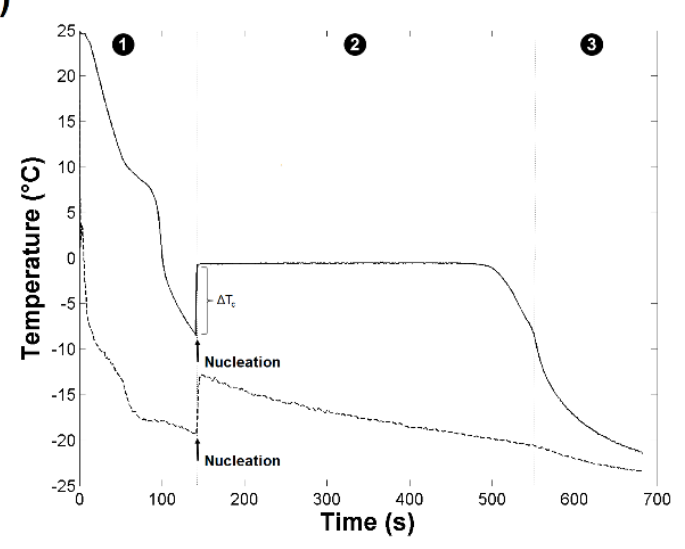


561
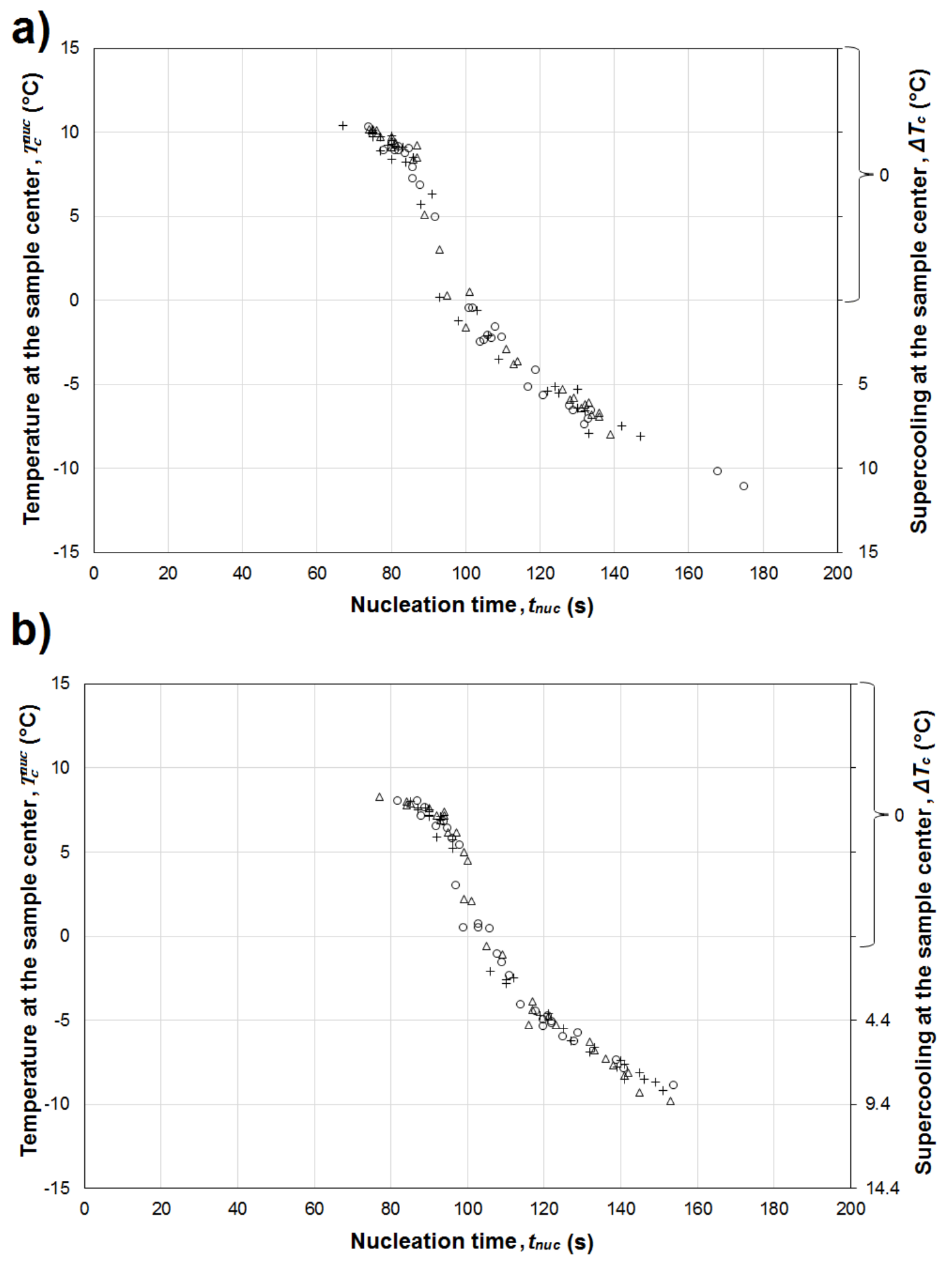
565

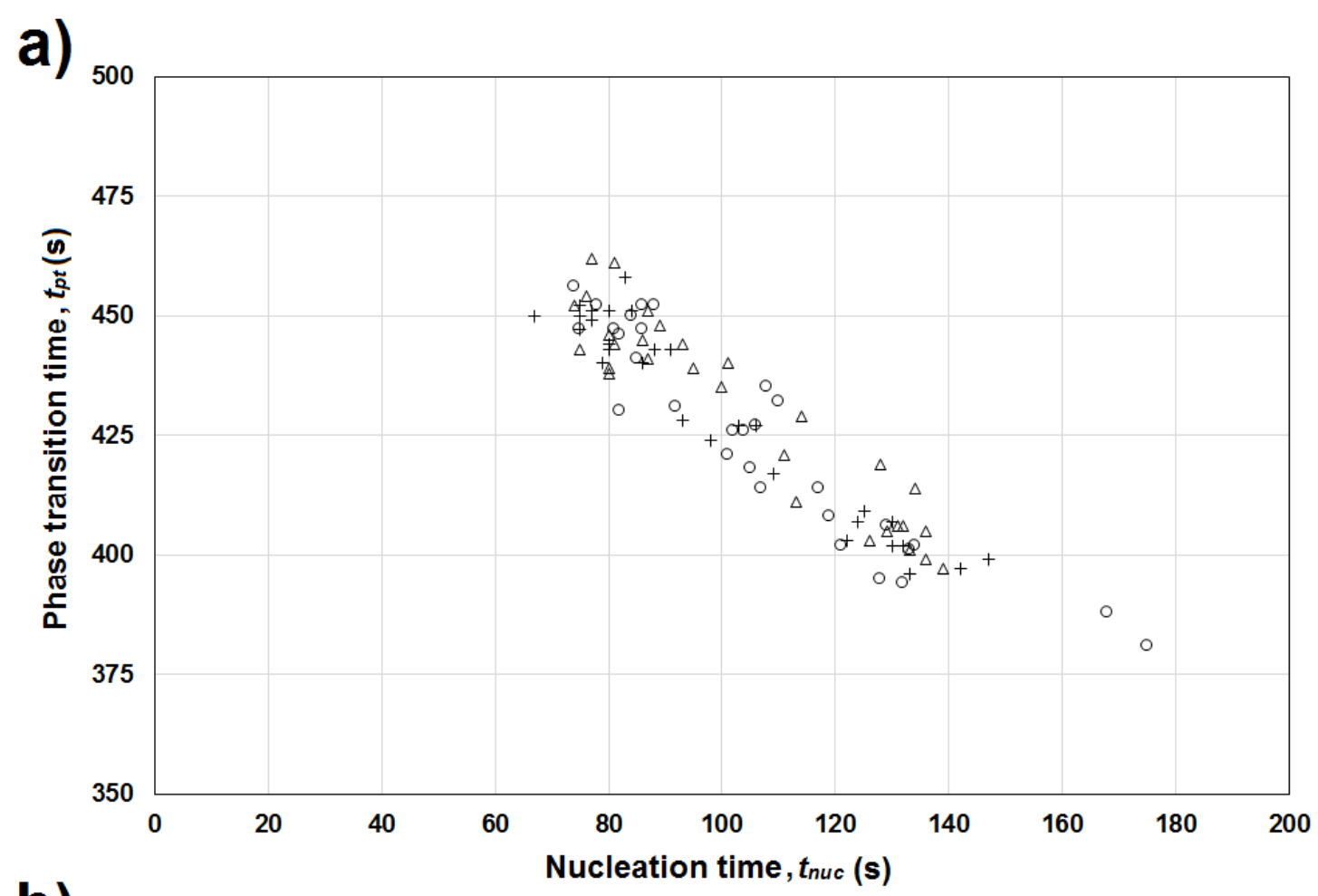

b)

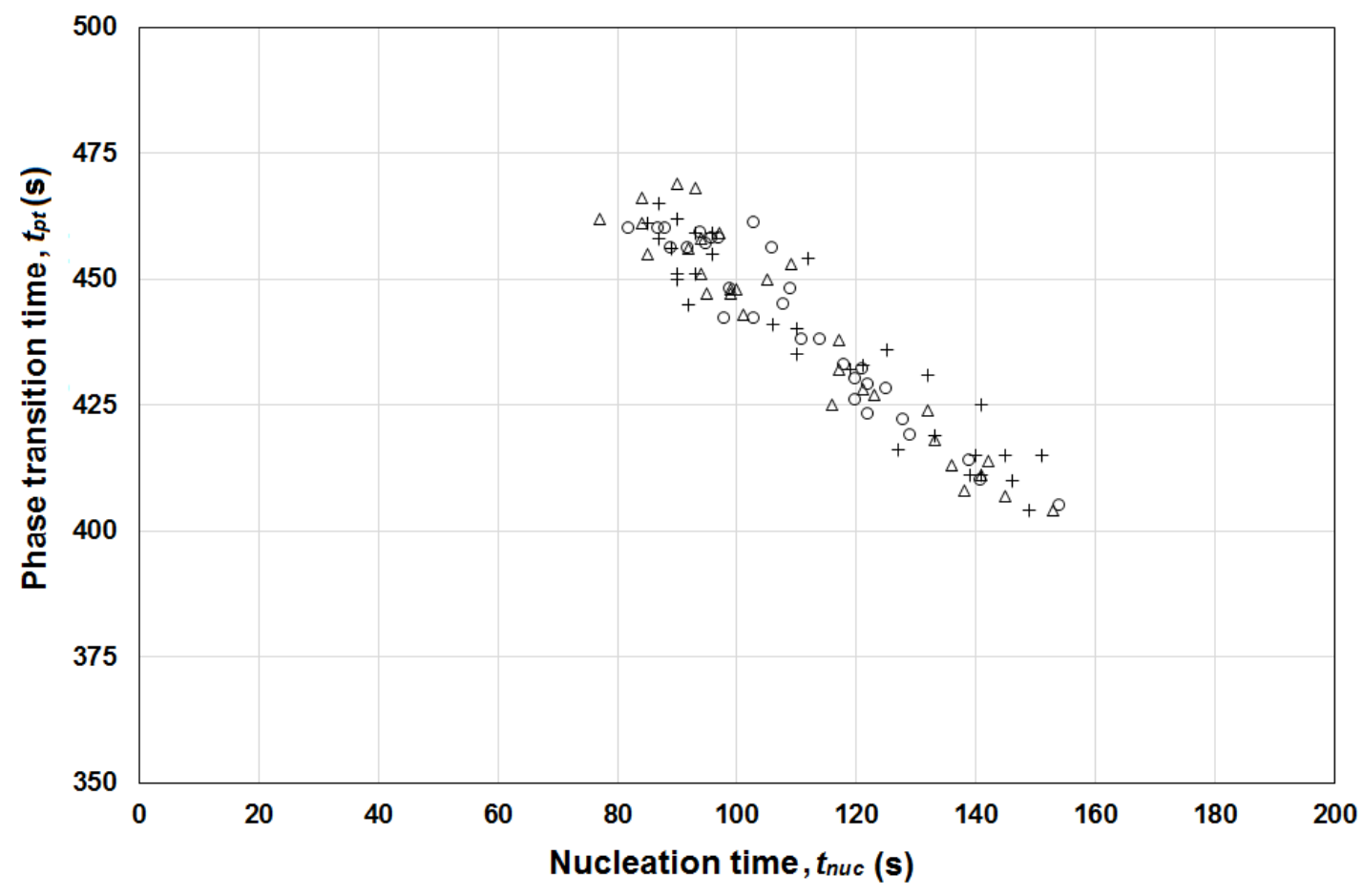

